\title{
Correlation energies of inhomogeneous many-electron systems
}

\author{
John F. Dobson ${ }^{1,2}$, Jun Wang ${ }^{1}$ and Tim Gould ${ }^{1}$ \\ ${ }^{1}$ Research Centre for Modelling and Computation, School of Science, Griffith University, Nathan, Queensland 4111, Australia \\ ${ }^{2}$ Groupe de Physique des Solides, Université de Paris, 2 Place Jussieu, 75251 Paris, France
}

(November 23, 2001)

We generalize the uniform-gas correlation energy formalism of Singwi, Tosi, Land and Sjölander to the case of an arbitrary inhomogeneous many-particle system. For jellium slabs of finite thickness with a self-consistent LDA groundstate Kohn-Sham potential as input, our numerical results for the correlation energy agree well with diffusion Monte Carlo results. For a helium atom we also obtain a good correlation energy.

71.10.-w,71.15.-m,73.21.-b,31.25.-v

Despite eighty years of study, the accurate calculation of the correlation energy of interacting quantal manyelectron systems is still often a challenge, even for some systems not regarded as "strongly correlated". For realistic many-electrons systems the current state of the art includes diffusion/Green function quantum Monte Carlo (DMC), variational and quantum chemical methods such as the Configuration Interaction (CI) approach. These all have stringent practical limitations to relatively small and/or not-too-complex systems. Approximate density functionals of the local-density (LDA) and generalized gradient (GGA) class [1] are in principle less accurate than the above approaches, but they remain feasible even for very large, complex systems, and often provide useful accuracy. They fail completely, however, to describe long-ranged correlations in cases where these differ qualitatively from those of the homogeneous electron gas. A case in point is the van der Waals (vdW) or dispersion interaction: all LDA/GGA approaches miss its long-ranged part completely [2] [3] [4], and give at best patchy results at short range [5], [6]. Thus DMC, CI and standard DFT methods are all likely to be problematical for large complex vdW systems of practical interest, including many soft-matter cases.

Here we present a new general approximation method ("ISTLS") for the correlation energy of inhomogeneous electronic systems, which we believe will be appropriate for vdW problems among others. It employs a selfconsistent scheme for the pair distribution based on the surprisingly accurate homogeneous-gas correlation energy method of Singwi, Tosi, Land and Sjolander (STLS) [7]. We take as input an approximate Kohn-Sham (KS) potential $v_{K S}(\vec{r})$ of the inhomogeneous system, and produce the correlation energy $E_{c}$ as output. Tractable input theories such as LDA or exchange-only Krieger-Li-Iafrate [8], by themselves, make large correlation-energy errors of order $+100 \%$ and $-100 \%$ respectively, yet starting from their KS potentials our ISTLS theory yields correlation energies accurate to a few percent. In its numerical complexity, and also in its accuracy for $E_{c}$ of large systems, ISTLS appears to be intermediate between LDA/GGA and the more microscopic approaches mentioned above.

Our original motivation for deriving ISTLS was primarily to address soft-matter problems such as polymer cohesion or the energetics of graphite and its intercalates. For such vdW systems, the RPA-like [3] nature of the method, together with its uniquely self-consistent localfield correction, suggests it will accurately describe $\mathrm{vdW}$ interactions [2] while also correctly treating other kinds of bonding 任. The method is by no means restricted to vdW problems, however, and should provide a useful alternative approach both for soft and for hard matter. In particular it appears to be highly competitive with recently-proposed correlation energy theories based on the GW method [9] ; it gives a much more accurate correlation energy in the homgeneous gas at large $r_{s}$, for example. Being intrinsically approximate, ISTLS needs to be tested. Therefore, in the present paper, we benchmark our method against state-of-the-art results for two simple but highly inhomogeneous situations: finite-thickness jellium slabs and the He atom. The results suggest that our scheme, while based on approximations known to work well in uniform electron gases, also gives a good treatment of strong inhomogeneity in one to three space dimensions. Details follow.

The groundstate energy of an inhomogeneous many electron system with external potential $v^{e x t}(\vec{r})$ and groundstate electron density $n(\vec{r})$ is given exactly by the constant-density adiabatic connection formula of formal Kohn-Sham density functional theory: 10,11

$$
\begin{aligned}
E_{0}= & T_{s}[n]+\int n(\vec{r}) v^{e x t}(\vec{r}) d \vec{r} \\
& +\frac{1}{2} \int \frac{e^{2}}{\left|\vec{r}-\vec{r}^{\prime}\right|} n(\vec{r}) n\left(\vec{r}^{\prime}\right) d \vec{r} d \vec{r}^{\prime}+E_{x c}[n] \\
E_{x c}[n]= & \frac{1}{2} \int_{0}^{1} d \lambda \int \frac{e^{2}}{\left|\vec{r}-\vec{r}^{\prime}\right|}\left(n_{2 \lambda}\left(\vec{r}, \vec{r}^{\prime}\right)-n(\vec{r}) n\left(\vec{r}^{\prime}\right)\right) d \vec{r} d \vec{r}^{\prime}
\end{aligned}
$$

Here $T_{s}[n]=\hbar^{2}(2 m)^{-1} \sum_{k o c c} \int\left|\nabla \phi_{k}(\vec{r})\right|^{2} d \vec{r}$ is the $\mathrm{KS}$ kinetic energy, and $\left\{\phi_{k}\right\}$ are the occupied KS orbitals, eigenfunctions of the one-electron KS potential $v_{K S}(\vec{r})$. 
$v_{K S}$ is defined to be such that independent electrons moving in $v_{K S}$ yield the true groundstate density:

$$
v_{K S} \Longrightarrow\left\{\phi_{k}(\vec{r})\right\}: n(\vec{r})=\sum_{k o c c}\left|\phi_{k}(\vec{r})\right|^{2} .
$$

The $\lambda$ integration in (3) accounts for the kinetic part of the KS correlation energy. The groundstate pair distribution $n_{2 \lambda}\left(\vec{r}, r^{\prime}\right)$ is that of a " $\lambda$-system"defined to have a reduced electron-electron interaction $\lambda e^{2} / r_{12}$, and a modified external potential $v_{\lambda}^{\text {ext }}(\vec{r})$ chosen to maintain the true $(\lambda=1)$ groundstate density at any $\lambda: n_{\lambda}(\vec{r})=n_{\lambda=1}(\vec{r}) \equiv$ $n(\vec{r})$. Remarkably [10, 11, only the true external potential $v^{e x t} \equiv v_{\lambda=1}^{e x t}$ appears in (便). Note that, from (4), the KS potential of each $\lambda$-system is the same as that of the true $(\lambda=1)$ system, because the density is the same:

$$
v_{K S, \lambda} \equiv v_{K S} .
$$

The groundstate pair distribution $n_{2 \lambda}$ in (3) can be related to the Kubo density-density response function $\chi_{\lambda}$ of the $\lambda$-system by the $T=0 K$ fluctuation-dissipation theorem [12, 13],

$$
\begin{aligned}
& n(\vec{r}) n\left(\vec{r}^{\prime}\right)\left(g_{\lambda}\left(\vec{r}, \vec{r}^{\prime}\right)-1\right) \equiv n_{2 \lambda}\left(\vec{r}, \vec{r}^{\prime}\right)-n(\vec{r}) n\left(\vec{r}^{\prime}\right) \\
= & -\frac{\hbar}{\pi} \int_{0}^{\infty} \chi_{\lambda}\left(\vec{r}, \vec{r}^{\prime}, \omega=i u\right) d u-n(\vec{r}) \delta\left(\vec{r}-\vec{r}^{\prime}\right) .
\end{aligned}
$$

Eq. (6) also introduces the equilibrium pair correlation factor $g_{\lambda}\left(\vec{r}, \vec{r}^{\prime}\right)$.

The Kohn-Sham density-density response $\chi_{K S}$ 114 is defined to be that of independent electrons moving in the KS potential $v_{K S}$. Note that, by (5),

$$
\chi_{K S, \lambda} \equiv \chi_{K S} \equiv \chi_{\lambda=0} .
$$

$\chi_{K S}$ is exactly expressible 14 by perturbation theory in terms of the KS orbitals $\left\{\phi_{k}\right\}$. From this expression it is readily shown that, when $\chi_{\lambda}$ is replaced by $\chi_{\lambda=0} \equiv$ $\chi_{K S}$, Eq. (8) gives the "exact DFT exchange" energy, i.e. it gives the Hartree-Fock energy integral in which the self-consistent Hartree-Fock orbitals are replaced by the KS orbitals $\phi_{k}$. Thus this formalism easily deals with exchange. Subtracting this DFT exchange energy from (6) we obtain the exact DFT correlation energy

$$
E_{c}=\frac{-\hbar}{2 \pi} \int d \vec{r} d \vec{r}^{\prime} \frac{e^{2}}{\left|\vec{r}-\vec{r}^{\prime}\right|}
$$

$\times \int_{0}^{1} d \lambda \int_{0}^{\infty} d u\left[\chi_{\lambda}\left(\vec{r}, \vec{r}^{\prime}, \omega=i u\right)-\chi_{\lambda=0}\left(\vec{r}, \vec{r}^{\prime}, \omega=i u\right)\right]$.

At each $\lambda$ value the interacting and KS response are related exactly by a Dyson-like screening integral equation 114] $\chi_{\lambda}=\chi_{\lambda=0}+\chi_{\lambda=0} *\left(\lambda V_{\text {coulomb }}+f_{x c \lambda}\right) * \chi_{\lambda}$, where spatial convolution is represented by a star. The xc kernel $f_{x c \lambda}$ contains the many-body xc effects and has tradionally been treated by a local density approximation [14]
[15] [16. Here, however, instead of using a local uniformgas-based approximation we effectively generate a nolocal $f_{x c}$ self-consistently for the particular inhomogeneous system. To do this we extend to nonuniform systems the semiclassical approach of STLS. Thus we relate the independent-electron and interacting responses by solving the time evolution equation (first BBGKY hierarchy equation [17]) for the one-electron distribution function $f(\vec{r}, \vec{p}, t)$ of the classical $\lambda$-system:

$$
\begin{aligned}
& \left(\frac{\partial}{\partial t}+m^{-1} \vec{p} \cdot \frac{\partial}{\partial \vec{r}}+\vec{F}_{\lambda}^{e x t}(\vec{r}, t) \cdot \frac{\partial}{\partial \vec{p}}\right) f(\vec{r}, \vec{p}, t) \\
= & \int\left(\frac{\partial}{\partial \vec{r}} \frac{\lambda e^{2}}{\left|\vec{r}-\vec{r}^{\prime}\right|}\right) \cdot \frac{\partial}{\partial \vec{p}} f_{\lambda}^{(2)}\left(\vec{r}, \vec{p} ; \vec{r}^{\prime}, \vec{p}^{\prime}, t\right) d \vec{r}^{\prime} d \vec{p}^{\prime} .
\end{aligned}
$$

This equation is exact but requires the dynamic pair distribution $f_{\lambda}^{(2)}$. The essential contribution of STLS was to use the equilibrium pair-density factor $g_{\lambda}\left(\vec{r}, \vec{r}^{\prime}\right)$ of Eq. (6) in a semi-classical truncation scheme

$$
f_{\lambda}^{(2)}\left(\vec{r}, \vec{p} ; \vec{r}^{\prime}, \vec{p}^{\prime}, t\right) \approx g_{\lambda}\left(\vec{r}, \vec{r}^{\prime}\right) f(\vec{r}, \vec{p}, t) f\left(\vec{r}^{\prime}, \vec{p}^{\prime}, t\right),
$$

where the true dynamic correlation factor $g_{\lambda}$ should depend on both the momenta and the time, but this dependence is ignored and $g_{\lambda}$ is taken to be the static, momentum-independent equilibrium density correlating factor from Eq. (6). Using (10) in (9) and linearizing about the equilibrium distribution, $f=f_{0}(\vec{r}, \vec{p})+$ $\delta f(\vec{r}, \vec{p}, t)$, we obtain a closed one-body kinetic equation

$$
\begin{aligned}
& \left(\frac{\partial}{\partial t}+m^{-1} \vec{p} \cdot \frac{\partial}{\partial \vec{r}}+\vec{F}^{(0)}(\vec{r}) \cdot \frac{\partial}{\partial \vec{p}} \cdot\right) \delta f(\vec{r}, \vec{p}, t) \\
= & -\delta \vec{F}^{e f f}(\vec{r}, t) \cdot \frac{\partial f_{0}(\vec{r}, \vec{p})}{\partial \vec{p}} .
\end{aligned}
$$

Here

$$
\vec{F}^{(0)}(\vec{r})=\vec{F}_{0 \lambda}^{e x t}(\vec{r})-\int\left(\frac{\partial}{\partial \vec{r}} \frac{\lambda e^{2}}{\left|\vec{r}-\vec{r}^{\prime}\right|}\right) g_{\lambda}\left(\vec{r}, \vec{r}^{\prime}\right) n_{0}\left(\vec{r}^{\prime}\right) d \vec{r}^{\prime}
$$

corresponds to the gradient of the KS potential in the quantal case, and is independent of $\lambda$ by choice of $F_{0 \lambda}^{e x t}(\vec{r})$. Further,

$$
\begin{aligned}
\delta \vec{F}^{e f f}(\vec{r}, t) & =\delta \vec{F}^{e x t}(r, t)+\int \vec{W}_{\lambda}\left(\vec{r}, \vec{r}^{\prime}\right) \delta n\left(\vec{r}^{\prime}, t\right) d \vec{r}^{\prime} \\
\vec{W}_{\lambda}\left(\vec{r}, \vec{r}^{\prime}\right) & =g_{\lambda}\left(\vec{r}, \vec{r}^{\prime}\right) \frac{-\partial}{\partial \vec{r}} \frac{\lambda e^{2}}{\left|\vec{r}-\vec{r}^{\prime}\right|} .
\end{aligned}
$$

(8) Because (11) is linear and time invariant, its solution $\delta n(\vec{r}, t) \equiv \int \delta f(\vec{r}, \vec{p}, t) d \vec{p}$ can be expressed in the form

$$
\delta n(\vec{r}, t)=\int \vec{\nu}_{0}\left(\vec{r}, \vec{r}^{\prime}, t-t^{\prime}\right) \cdot \delta \vec{F}^{e f f}\left(\vec{r}^{\prime}, t^{\prime}\right) d \vec{r}^{\prime} d t^{\prime}
$$

where a $\vec{\nu}_{0}$ is a $\lambda$-independent classical vector response function giving the independent-electron density response to an applied force, with $\left(\partial / \partial \vec{r}^{\prime}\right) \cdot \vec{\nu}_{0}=\chi_{\lambda=0}$. 
In the case of homogeneous electron gases, $\vec{F}_{0}$ is zero and $g_{\lambda}$ is a function only of the separation $R \equiv\left|\vec{r}-\vec{r}^{\prime}\right|$, and then $\vec{\nabla} \times \vec{W}=0$ so that the effective pair force $\vec{W}_{\lambda}$ is irrotational and can be expressed as a gradient of a scalar potential, $\vec{W}_{\lambda}(\vec{R})=-(\partial / \partial \vec{R}) w_{\lambda}(R)$. Then, assuming $\delta \vec{F}^{e x t}$ comes from a potential $\delta V^{e x t}$ we can use integration by parts (Green's theorem) followed by space Fourier transformation to write (14) in $q$-space as $\delta n=\chi_{\lambda=0} \delta V^{e f f}=\chi_{\lambda=0}\left(\delta V^{e x t}+w_{\lambda} \delta n\right)$. This yields $\chi_{\lambda}(q, \omega)=\chi_{\lambda=0}(q, \omega)\left(1-w_{\lambda}(q) \chi_{\lambda=0}(q, \omega)\right)^{-1}$. This equation resembles a classical Random Phase Approximation (RPA), with $w_{\lambda}(q)$ replacing the bare coulomb pair potential $4 \pi \lambda e^{2} / q^{2}$. This response $\chi_{\lambda}$ depends on $g_{\lambda}$ via $w_{\lambda}$, and $g_{\lambda}$ is determined by $\chi_{\lambda}$ via (6), giving a closed selfconsistent scheme. STLS applied this theory to the degenerate electron gas by replacing the classical Boltzmann-equation density response $\chi_{\lambda=0}$ with the quantal Lindhard response. Despite the crudeness of the factorization (10), the STLS formalism gives excellent correlation energies for both $3 \mathrm{D}$ and $2 \mathrm{D}$ homogeneous electron gases, up to relatively large values of the inter-electron spacing parameter $r_{s}$. For example, in 3D $E_{c}^{S T L S}$ is within about $1 \%$ of the 3D diffusion Monte Carlo results [18] for $2<r_{s} \leq 5$. The error is still under $4 \%$ at $r_{s}=20$ and under $7 \%$ at $r_{s}=50$, a regime including gases generally regarded as significantly correlated. These results are significantly better than, e.g. recent GW-based many-body methods [9] which give $20 \%$ error at $r_{s}=20$. The homogeneous STLS scheme has a number of shortcomings including unphysical negative values of the on-top pair factor $g(\vec{r}, \vec{r})$ and failure to satisfy the compressibility sum rule. Further work addressing these difficulties [19], [20], 21], [22], [23] did not, however, significantly improve the predicted uniform egas correlation energy. Therefore in the present work we have concentrated on generalizing the original semi-classical STLS scheme to inhomogeneous systems. This does not appear to have been attempted previously: bilayered electron gases have certainly been treated [24], but these are isomorphic to a two-species homogeneous $2 \mathrm{D}$ electron gas. We will show that the formalism is tractable for cases of genuine inhomogeneity.

In an inhomogeneous system we have $\vec{\nabla} \times \vec{W}_{\lambda}=$ $-\vec{\nabla} g_{\lambda}\left(\vec{r}, \vec{r}^{\prime}\right) \times \vec{\nabla}\left(\lambda e^{2} /\left|\vec{r}-\vec{r}^{\prime}\right|\right) \neq \overrightarrow{0}$ so that there is no scalar potential corresponding to $\vec{W}_{\lambda}$, and the vector bare response $\vec{\nu}_{0}$ from Eq. (14) must be used: the scalar version $\chi_{0}$ is not sufficient. This is an essential difference between the inhomogeneous case and the homogeneous one. As in the homogeneous case, we postulate that a degenerate Fermi system can be treated via the above semi-classical analysis by using the quantal-Fermi independent-electron response for $\vec{\nu}_{0}$ : this also amounts to using the quantal KS potential in place of its classical counterpart $\vec{F}_{0}$. By perturbation of the occupied independent-electron (Kohn-Sham, KS) orbitals $\phi_{j}(\vec{r})$, we obtained for the inhomogeneous quantal response at imaginary frequency $i u$

$$
\begin{aligned}
\vec{\nu}_{0}\left(\vec{r}, \vec{r}^{\prime}, \omega\right. & =i u)=\frac{1}{u} \operatorname{Re}\left[\frac{i \hbar}{m} \sum_{j} f_{j} \phi_{j}^{*}(\vec{r})\right. \\
\times\left[G \left(\vec{r}, \vec{r}^{\prime}, E\right.\right. & \left.=\hbar \omega_{j}+i \hbar u\right) \vec{\nabla}^{\prime} \phi_{j}\left(\vec{r}^{\prime}\right) \\
-\phi_{j}\left(\vec{r}^{\prime}\right) \vec{\nabla}^{\prime} G\left(\vec{r}, \vec{r}^{\prime}, E\right. & \left.\left.=\hbar \omega_{j}+i \hbar u\right)\right]
\end{aligned}
$$

where $f_{j}$ is the Fermi occupation factor and $G$ is the Green function for a single electron moving in the groundstate Kohn-Sham potential $v_{K S}(r)$. The Coulomb screening conditions (12), (13), (14) for the inhomogeneous case can be written as a Dyson-like "screening" integral equation for the interacting response $\chi_{\lambda}$ :

$$
\begin{gathered}
\chi_{\lambda}\left(\vec{r}, \vec{r}^{\prime}, \omega\right)=\chi_{K S}\left(\vec{r}, r^{\prime}, \omega\right)+\int Q_{\lambda}\left(\vec{r}, \vec{r}^{\prime \prime}, \omega\right) \chi_{\lambda}\left(\vec{r}^{\prime \prime}, \vec{r}^{\prime}, \omega\right) d r^{\prime \prime}, \\
Q_{\lambda}\left(\vec{r}, \vec{r}^{\prime \prime}, \omega\right)=\int \vec{\nu}_{0}\left(\vec{r}, \vec{r}^{\prime \prime \prime} \omega\right) . \vec{W}_{\lambda}\left(\vec{r}^{\prime \prime \prime}, \vec{r}^{\prime \prime}\right) d \vec{r}^{\prime \prime \prime}, \chi_{K S}=\vec{\nabla}^{\prime} . \vec{\nu}_{0}
\end{gathered}
$$

We term this the "inhomogeneous STLS" (ISTLS) scheme. To demonstrate its feasibility and accuracy we have carried it out numerically for two highly inhomogeneous but spatially symmetric cases, namely (i) chargeneutral jellium slabs and (ii) a helium atom.

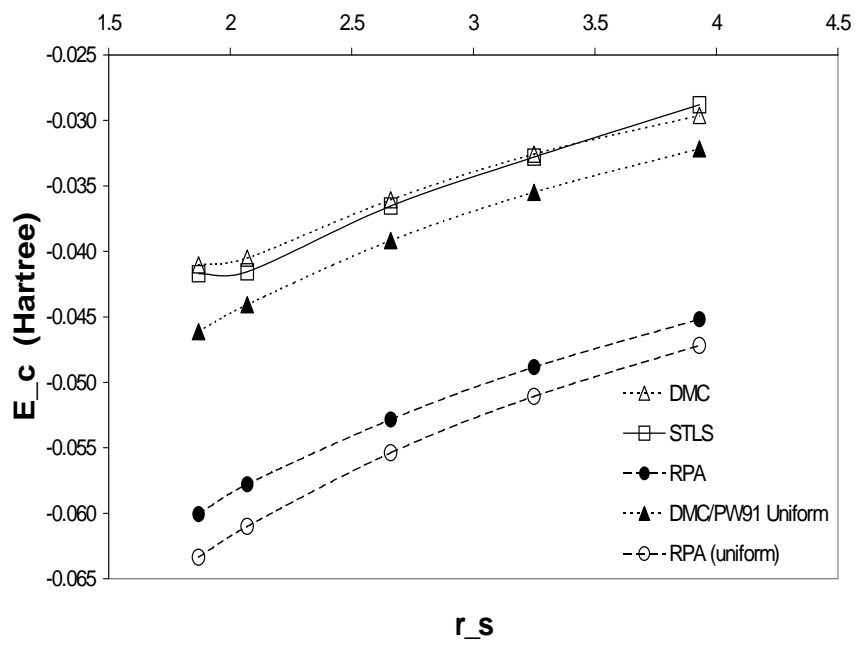

FIG. 1. Correlation energy of neutral jellium slabs (Hartree/el)

The jellium slabs were first solved in the LDA (LDA-Perdew-Wang 1991 [25]) to give the selfconsistent groundstate Kohn-Sham potential $v_{K S}^{L D A}(z)$ and density $n(z)$, where $z$ is the space coordinate in the thin dimension of the slab. Our ISTLS formalism was applied as a "post-functional" giving the correlation energy starting from the fixed $v_{K S}^{L D A}(z)$, though of course ideally one would choose a $v(z)$ to minimize the total energy including the STLS corrections. (This OPM method will also 
give an improved density $n(\vec{r}))$. Eqs. (6), (13) and (15) - (17) were then implemented. To perform the iterative refinement of the static pair factor $g_{\lambda}\left(r, r^{\prime}\right)$, we started with a $g$ of Hartree-Fock form based on the occupied KS orbitals from the LDA groundstate. Finally (8) was used.

Fig. 1 gives the slab correlation energy per electron (open symbols) for a number of positive background densities $n_{0+}$, parametrized by the dimensionless interelectron spacing $r_{s}=m e^{2} \hbar^{-2}\left(3 n_{0+} / 4 \pi\right)^{1 / 3}$. We show results from ISTLS (solid line), DMC [26] (dotted line) and RPA (dashed line) schemes, the last obtained by setting the pair correlation factor $g$ to unity in Eqs. (13) and (15) - (17). The thickness of the positive background in each slab is $L=7.21 r_{s} a_{B}$, to match the available DMC results. Results per electron in the unbounded uniform gas are also shown (closed symbols). Agreement of $E_{c}^{I S T L S}$ with the slab DMC data is good, within $3 \%$. This is comparable to the agreement of STLS with DMC for the uniform 3D gas with $2 \leq r_{s} \leq 20$.

For small finite systems such as atoms one needs a self-interaction correction (SIC) in the starting KS potential and density. Otherwise (as for example when one uses the simple LDA) unrealistic response functions are obtained because the asymptotic $-e^{2} / r$ potential is missing in $v^{K S}$. We solved helium using the KriegerLi-Iafrate exchange-only description [8] of the atomic groundstate. This has the advantage of a common potential $v^{K L I} \equiv v^{K S}$ for all orbitals. The explicit spherical form of Eqs. (15) - (17) involves spherical harmonics but is somewhat cumbersome because of the vector character of $\vec{\nu}_{0}$. We obtained a total ISTLS He correlation energy of -40.0 milliHartree, within $5 \%$ of the "exact" nonrelativistic value [28] of $-42.0 \mathrm{mH}$. Our result is of "chemical accuracy". The KLI starting potential is adequate: we re-ran our method starting from the numerically exact He KS potential [27], obtaining $<<1 \%$ change in $E_{c}$.

In summary, we have derived an inhomogeneous generalization ("ISTLS") of the rather successful STLS uniform-gas correlation energy formalism: see Eqs. 113), (15), (17), (16), (6), and (8). We have shown that ISTLS gives good groundstate correlation energies in some highly inhomogeneous electronic systems. The scheme can also encompass finite temperatures, and plasmon calculations. An advantage of the ISTLS scheme is that its pair correlation physics is "self-tailored" to the system at hand, rather than "stolen" from some reference system as in some density functional schemes, or guessed then optimized as in variational schemes. It does this, moreover, without the need for solving explicit two-body dynamic equations. We speculate that interesting density functionals for the correlation energy might be derived by using the ISTLS scheme with semi-local-density approximations for the bare response $\vec{\nu}_{0}$, somewhat as in [3].

JFD acknowledges an Australian Research Council Large grant and the hospitality of Prof. M. Combescot,
Université de Paris VII. The atomic application of ISTLS forms part of the PhD thesis of TG. We thank Cyrus Umrigar for exact atomic $v_{K S}$ data.

[1] W. Kohn, A. Becke, and R. G. Parr, J. Phys. Chem. 100, 12974 (1996).

[2] W. Kohn, Y. Meir, and D. E. Makarov, Phys. Rev. Lett. 80, 4153 (1998).

[3] J. F. Dobson and J. Wang, Phys. Rev. Lett. 82, 2123 (1999).

[4] J. F. Dobson et al., Australian J. Chem. 54, 513 (2001).

[5] D. C. Patton and M. R. Pederson, Phys. Rev. A 56, R2495 (1997).

[6] S. Tsuzuki and H. P. Luthi, J. Chem. Phys. 114, 3949 (2001).

[7] K. S. Singwi, M. P. Tosi, R. H. Land, and A. Sjolander, Phys. Rev. 176, 589 (1968).

[8] Y. Li, J. B. Krieger, and G. J. Iafrate, Phys. Rev. A 47, 165 (1993).

[9] P. Garcia-Gonzalez and R. W. Godby, Phys. Rev. Lett. 88, 056406 (2002): Phys. Rev. B 63, 075112 (2001).

[10] D. C. Langreth and J. P. Perdew, Sol. State Commun. 17, 1425 (1975).

[11] O. Gunnarsson and B. I. Lundqvist, Phys. Rev. B 13, 4274 (1976).

[12] L. D. Landau and E. Lifshitz, Statistical Physics (Addison-Wesley, Reading, Massachusetts, 1969).

[13] J. F. Dobson, in Electronic Density Functional Theory: Recent Progress and New Directions, edited by J. F. Dobson, G. Vignale, and M. P. Das (Plenum, New York, 1998), p. 43.

[14] E. K. U. Gross and W. Kohn, Phys. Rev. Lett. 55, 2850 (1985).

[15] G. Vignale and W. Kohn, Phys. Rev. Lett. 77, 2037 (1996).

[16] J. F. Dobson and J. Wang, Phys. Rev. B 62, 10038 (2000).

[17] T. J. M. Boyd and J. J. Sanderson, Plasma Dynamics (Barnes and Noble, New York, 1969).

[18] D. M. Ceperley and B. J. Alder, Phys. Rev. Lett. 45, 566 (1980).

[19] H. Hayashi and M. Shimizu, J. Phys. Soc. Jap. 48, 16 (1980).

[20] K. S. Singwi, A. Sjölander, M. P. Tosi, and R. H. Land, Phys. Rev. B 1, 1044 (1970).

[21] T. Hasegawa and M. Shimizu, J. Phys. Soc. Jap. 38, 965 (1975).

[22] A. Holas and S. Rahman, Phys. Rev. B 35, 2720 (1987).

[23] P. Vashishta and K. S. Singwi, Phys. Rev. B 6, 875 (1972).

[24] J. Szymanski, L. Swierkowski, and D. Neilson, Phys. Rev. B 50, 11002 (1994).

[25] J. P. Perdew and Y. Wang, Phys. Rev. B 45, 13244 (1992).

[26] P. H. Acioli and D. M. Ceperley, Phys. Rev. B 54, 17199 (1996).

[27] C. J. Umrigar and X. Gonze, Phys. Rev. A 50, 3827 (1994).

[28] S. J. Chakravorty et al., Phys. Rev. A 47, 3649 (1993). 